\title{
The first supramolecular consideration on crystal of DFMO - promising antiviral and anticancer pan-drug
}

\author{
Joanna Bojarska \\ Department of Chemistry, Technical University of Lodz, Poland \\ joanna.bojarska@p.lodz.pl
}

Targeting the polyamine biosynthetic pathway by inhibiting ornithine decarboxylase (ODC) is a powerful approach in the fight against both viruses and cancers.

$D, L$-alpha-ifluoromethylornithine (also called DFMO, eflornithine, ornidyl) is the best-known inhibitor of ODC and a broad-spectrum, unique therapeutical agent. It is promising pan-drug against diverse cancers, such as leukemia, skin cancer, breast cancer, prostate cancer and pancreatic cancer, cervical, small-cell lung cancer and melanoma, gastric cancer, colorectal cancer, neuroblastoma, glial tumors, such as malignant gliomas, as well as antiviral pan-drug, against RNA and DNA viruses, inter alia against dengue virus, zika, chikungunya virus, hepatitis B virus, human cytomegalovirus, herpes simplex virus, coxsackievirus B3, ebola, hepatitis C virus, sindbis virus, Japanese encephalitis virus, yellow fever virus, enterovirus 71, polio, rift valley fever virus, vesicular stomatitis virus, rabies virus, la crosse virus, semliki forst virus, as well as Middle East respiratory syndrome coronavirus (MERS-CoV) and severe acute respiratory syndrome coronavirus 2 (SARS-CoV-2).

Here, we present the first supramolecular study focusing on the structure of DFMO. We discuss qualitative and quantitative survey of non-covalent interactions via Hirshfeld surface, molecular electrostatic potential, enrichment ratio and energy frameworks analysis visualizing 3-D topology of interactions in order to understand the differences in the cooperativity of interactions involved in the formation of supramolecular synthons at the subsequent levels of well-organized supramolecular self-assembly, in comparison with the ornithine structure.

In the light of the drug discovery, supramolecular studies of amino acids, essential constituents of proteins, are of prime importance. In brief, the same amino-carboxy synthons are observed in the bio-system containing DFMO.

1. Bojarska J, New R, Borowiecki P, Remko M, Breza M, Madura ID, Fruzi 'nski A, Pietrzak A and Wolf WM (2021) The First Insight Into the Supramolecular System of D,L $\alpha$-Difluoromethylornithine: A New Antiviral Perspective. Front. Chem. 9:679776. doi: 10.3389/fchem.2021.679776

Keywords: modified amino acids; pandemic; antivirals 